\title{
Predicción de crecientes incorporando información histórica con base en la distribución log-normal de 3 parámetros
}

\author{
Flood Predictions Incorporating Historic Information \\ Through the 3 Parameters Log-Normal Distribution
}

\author{
Campos-Aranda D.F. \\ Facultad de Ingeniería \\ Universidad Autónoma de San Luis Potosí \\ E-mail:campos_aranda@hotmail.com
}

Información del artículo: recibido: enero de 2007, aceptado: agosto de 2010

\section{Resumen}

Se destaca la importancia de la información histórica sobre crecientes, como complemento del registro sistemático, para garantizar que las estimaciones probabilísticas de las crecientes se ajusten a las experiencias de la comunidad y mejoren estadísticamente tales predicciones. Se expone con detalle el más simple de los métodos que incorporan información histórica en el estudio de crecientes, denominado de los momentos ponderados históricamente, incluyendo las ecuaciones que conducen a los tres parámetros de ajuste de la distribución log-normal. A continuación, se expone una fórmula de posición gráfica simple para llevar al papel de probabilidad este tipo de datos mezclados (históricos y sistemáticos). Posteriormente, se describen cuatro aplicaciones numéricas en ríos que cuentan con información histórica y reciente sobre crecientes, misma que ha sido expuesta en la literatura especializada. Por último, se formula una conclusión relativa al procedimiento descrito y a los resultados obtenidos.

\footnotetext{
Abstract

The importance of historic flood information is pointed out, as a complement to systematic records; oriented to guarantee the fit of probabilistic flood estimates to community experience and to improve statistically such predictions. The simplest method that incorporates historic information in flood studies is exposed in detail, named historically weighted moments, the equations to fit the three parameters in log-normal distribution are also listed. Then, a simple plotting positions formula is exposed, in order to show in probability paper this combined data (historic and systematic). Late, four numerical applications in rivers with historical and recent flood information are described, the information has previously been reported in technical papers. Finally, one conclusion is formulated relative to the process described and the numerical results obtained.
}

\section{Descriptores}

- predicción de crecientes

- crecientes históricas

- distribución Log-Normal

- método de momentos

\section{Keywords}

- flood predictions

- historic floods

- Log-Normal distribution

- moments method 


\section{Introducción}

Cuando se realiza un estudio hidrológico para estimación de crecientes o avenidas asociadas a determinadas probabilidades de excedencia, cuyo recíproco es el período de retorno, se debe de utilizar toda la información disponible, la cual puede ser: (1) registros sistemáticos, (2) datos históricos, (3) registros hidrométricos cercanos y (4) registros pluviográficos y pluviométricos para estimación indirecta. En México, la información sobre registros sistemáticos, comúnmente gastos instantáneos máximos anuales, ha sido digitalizada, concentrada y está disponible en el sistema BANDAS (IMTA, 2003).

En algunas localidades, principalmente donde la población ha ocupado planicies de inundación por largo tiempo, existe información acerca de las grandes crecientes que han ocurrido, antes o después del registro sistemático. Esta información se puede utilizar en el estudio de crecientes y frecuentemente define un periodo mayor durante el cual los gastos máximos históricos y sistemáticos son conocidos. La información histórica de crecientes se obtiene de informes que fueron elaborados para documentar tales eventos extraordinarios, así como del registro de noticias en periódicos y otras fuentes disponibles, como es el establecimiento de marcas o niveles máximos alcanzados por las inundaciones (Benson, 1968). El uso de los datos históricos garantiza que las estimaciones de crecientes se ajusten a las experiencias de la comunidad y mejora estadísticamente las predicciones (WRC, 1977).

El estudio convencional de crecientes basado en un registro sistemático, utiliza los gastos máximos anuales y por lo tanto, el tamaño de la muestra $n$ corresponde al número de años del registro, generalmente breve en comparación con las magnitudes de los periodos de retorno que se desean estimar. Entonces, cualquier información histórica que efectivamente aumente el valor de $n$, mejorará las estimaciones de crecientes. Aunque sólo se conozca el valor de una creciente histórica que alcanzó un cierto nivel, existe también el conocimiento que en el intervalo de años que no existe registro, los gastos máximos anuales fueron menores. Tal registro histórico puede ser considerado una muestra truncada o censurada, cuyos lapso y gasto umbral son conocidos (WRC, 1977; Condie et al., 1982).

\section{Momentos ponderados históricamente}

Quizás, el más simple de los métodos que incorporan información histórica en el estudio de crecientes sea el de los momentos ponderados históricamente (mph). En este método (WRC, 1977; Tasker et al., 1978; Condie et al., 1982) se comienza por definir el lapso total en años $(Y t)$ que abarcan los datos históricos y el registro sistemático, enseguida se calcula el factor de ponderación $W$, el cual es función de $Y t$ y del número de crecientes mayores $(n a)$ y menores $(n b)$ que el gasto umbral $(X c)$.

$W=(Y t-n a) / n b$

Ahora, siendo $x a$ y $x b$ las magnitudes de las crecientes superiores y menores que el gasto umbral, $y d a y d b$ las desviaciones de éstas con respecto de la media aritmética ajustada $\hat{x}$ (ecuación 2), entonces los mph son:

$$
\begin{aligned}
& \hat{x}=\left(W \sum_{1}^{n b} x b+\sum_{1}^{n a} x a\right) / Y t \\
& \hat{s}=\left[\left(W \sum_{1}^{n b} d b^{2}+\sum_{1}^{n a} d a^{2}\right) /(Y t-1)\right]^{1 / 2} \\
& \hat{g}=Y t\left[\left(W \sum_{1}^{n b} d b^{3}+\sum_{1}^{n a} d a^{3}\right) / \hat{s}^{3}\right] /[(Y t-1)(Y t-2)]
\end{aligned}
$$

\section{Ajuste de la distribución log-normal de 3 parámetros}

Con base en los mph los parámetros de ubicación $(\alpha)$, forma $(\mu)$ y escala $(\sigma)$ de la distribución Log-normal son (Condie et al., 1982):

$$
\begin{aligned}
& \alpha=\hat{x}-\hat{s} / c \\
& \mu=\ln (\hat{s} / c)-0.50 \ln \left(c^{2}+1\right) \\
& \sigma=\left[\ln \left(c^{2}+1\right)\right]^{1 / 2}
\end{aligned}
$$

siendo $c$ la raíz de la ecuación cúbica siguiente:

$c^{3}+3 c-\hat{g}=0$

cuya solución (Abramowitz et al., 1972) se obtiene haciendo:

$$
\begin{array}{ll}
r=\hat{g} / 2 & r c r=\sqrt{1+r^{2}} \\
s_{1}=[r+r c r]^{1 / 3} & s_{2}=[r-r c r]^{1 / 3}
\end{array}
$$

con

$c=s_{1}+s_{2}$ 
Las predicciones $(X p)$ buscadas, asociadas a una determinada probabilidad $(p)$ se obtienen mediante la expresión siguiente:

$X p=\alpha+\exp (\mu+K p \cdot \sigma)$

donde $K p$ es la desviación normal estándar que se estima con la aproximación siguiente (Abramowitz et al., 1972):

$f=\sqrt{\ln \left(1 / p^{2}\right)}$

$K p=f-\frac{c_{0}+c_{1} f+c_{2} f^{2}}{1+d_{1} f+d_{2} f^{2}+d_{3} f^{3}}$

con

$c_{0}=2.515517 \quad c_{1}=0.802853 \quad c_{2}=0.010328$

$d_{1}=1.432788 \quad d_{2}=0.189269 \quad d_{3}=0.001308$

cuando $0<p<0.50$, hacer $K p=-K p$; en caso de que $0.50<p<1.0$ emplear: $p=1-p$, sin cambiar Kp.

\section{Posiciones gráficas}

Las fórmulas de posiciones gráficas que permiten dibujar los datos en un papel de probabilidad, tienen dos funciones: primera, permitir realizar el contraste gráfico de la distribución ajustada y segunda, detectar valores dispersos o outliers. Cuando se desea incorporar la información histórica en la distribución empírica se deben realizar ajustes a las fórmulas tradicionales, uno de ellos, nuevamente el más simple, es el propuesto por el WRC (1977), otro más preciso es el sugerido por Hirsh et al. (1987). El primero consiste en ordenar en forma decreciente todos los gastos disponibles, históricos y sistemáticos y utilizar la expresión siguiente para estimar su probabilidad de excedencia:

$P(X>x)=\frac{m^{\prime}}{Y t+1}$

$m^{\prime}$ es el número de orden, igual a $m=1,2,3, \ldots, N A H$ o número de avenidas históricas. Cuando $m$ es mayor se corrige con base en las ecuaciones siguientes:

$m^{\prime}=W p \cdot m-(W p-1) \cdot(N A H+0.50)$

donde:

$W p=\frac{(Y t-N A H)}{n}$

siendo $n$ el número de datos del registro sistemático.

\section{Aplicaciones numéricas}

Río Júcar en la costa mediterránea de España

Con una superficie de cuenca de $22,000 \mathrm{~km}^{2}$, la parte media y baja de ésta son las que contribuyen a sus crecientes, las cuales han sido registradas sistemáticamente desde 1946 hasta 1988 por la estación 89 en el Huerto de Mulet (Francés, 1995), cuyos datos se tienen en la tabla 1. Respecto a las crecientes históricas se han documentado y verificado las cuatro siguientes en el periodo de 1792 a 1945, con gasto umbral de 6,200 m³ $/ \mathrm{s}: 1$ ) año 1805 con $\left.8,400 \mathrm{~m}^{3} / \mathrm{s}, 2\right)$ año 1814 con $6,400 \mathrm{~m}^{3} / \mathrm{s}, 3$ ) año

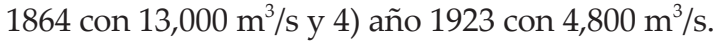

En la tabla 5 se presentan los valores característicos $(Y t, n a, n b, \hat{x}, \ldots, c)$ asociados al procesamiento de esta información, así como las predicciones obtenidas con la ecuación 9 y con la distribución Log-Pearson tipo III (LP3), modelo prescrito para datos sistemáticos.

\section{Río Big Sandy en Bruceton, TN., U.S.A.}

Con un área de cuenca de 530.7 km² la información histórica abarca de 1897 a 1929, con tres crecientes en los años inicial, 1919 y 1927, sus valores son 707.9, 594.7 y $523.9 \mathrm{~m}^{3} / \mathrm{s}$ (WRC, 1977). Los datos sistemáticos comprenden de 1930 a 1973 y se presentan en la tabla 2. Dado que en esta información no se especifica el valor de gasto umbral, se adoptó de $300 \mathrm{~m}^{3} / \mathrm{s}$. En la tabla 5 se han concentrado los resultados de este procesamiento y en la figura 1 se presenta su contraste gráfico.

\section{Río Irwell en Adelphi Weir, Inglaterra}

Se tiene un registro histórico de 10 crecientes superiores a $240 \mathrm{~m}^{3} / \mathrm{s}$ disponible en el periodo de 1896 a 1935 . Las mediciones sistemáticas comenzaron en 1936, sus datos anuales hasta 1968, con dos valores faltantes en 1940 y 1960, se tienen en la tabla 3 (NERC, 1975). Entonces, el registro histórico total es de 73 años, con 41 gastos de crecientes, más 32 crecientes truncadas (Condie et al., 1982). En la tabla 5 se presentan los resultados de su procesamiento y en la figura 2 se presenta su contraste gráfico.

\section{Río Avon en Bath, Inglaterra}

Durante el periodo histórico de 1865 a 1939 ocurrieron 10 crecientes superiores a $200 \mathrm{~m}^{3} / \mathrm{s}$. El registro sistemático disponible abarca de 1940 a 1968; ambas secuencias de datos se tienen en la tabla 4 (Leese, 1974). En la tabla 5 se citan los resultados asociados a su procesamiento y contraste. 


\section{Contraste contra predicciones del registro sistemático}

Con objeto de tener una comparación cuantitativa de las predicciones con el método de los $\mathbf{m p h}$, se realizó el ajuste a los datos sistemáticos de una distribución LogPearson tipo III, a través de los métodos de momentos en el dominio logarítmico (WRC, 1977) y en el dominio real (Bobée, 1975), adoptando el que condujo al menor error estándar de ajuste (Kite, 1977 y Campos, 2006). Los resultados se han concentrado en la tabla 5.

Se observa que en las tres primeras aplicaciones, la incorporación de la información histórica conduce a predicciones superiores a las obtenidas con el procesamiento probabilístico del registro sistemático; sin embargo, en la cuarta aplicación (Río Avon) sucede lo contrario, esto es debido a que en el registro reciente de sólo 29 años ocurren crecientes del mismo orden de magnitud que las históricas e incluso mayores, tal es el caso de los años 1960, 1967, 1954, 1946, 1963 y 1950 (tabla 4).

Tabla 1. Gastos sistemáticos en $\mathrm{m}^{3}$ /s del Río Júcar en la estación 89, España. (Francés, 1995)

\begin{tabular}{cc|cc|cc|cc}
\hline Año & Gasto & Año & Gasto & Año & Gasto & Año & Gasto \\
\hline 1946 & 464 & 1957 & 440 & 1968 & 161 & 1979 & 226 \\
1947 & 447 & 1958 & 800 & 1969 & 461 & 1980 & 87 \\
1948 & 880 & 1959 & 302 & 1970 & 133 & 1981 & 46 \\
1949 & 181 & 1960 & 114 & 1971 & 556 & 1982 & 12,000 \\
1950 & 860 & 1961 & 143 & 1972 & 377 & 1983 & 31 \\
1951 & 315 & 1962 & 207 & 1973 & 163 & 1984 & 347 \\
1952 & 93 & 1963 & 128 & 1974 & 70 & 1985 & 511 \\
1953 & 375 & 1964 & - & 1975 & 347 & 1986 & 413 \\
1954 & 134 & 1965 & 362 & 1976 & 319 & 1987 & 5,200 \\
1955 & 177 & 1966 & 161 & 1977 & 524 & 1988 & 207 \\
1956 & 954 & 1967 & 155 & 1978 & 87 & - & - \\
\hline
\end{tabular}

Tabla 2. Gastos sistemáticos en $\mathrm{m}^{3} / \mathrm{s}$ del Río Big Sandy en la estación Bruceton, U.S.A. (WRC, 1977)

\begin{tabular}{cc|cc|cc|cc}
\hline Año & Gasto & Año & Gasto & Año & Gasto & Año & Gasto \\
\hline 1930 & 257.7 & 1941 & 34.0 & 1952 & 120.6 & 1963 & 77.6 \\
1931 & 58.3 & 1942 & 286.0 & 1953 & 141.6 & 1964 & 87.8 \\
1932 & 221.4 & 1943 & 107.0 & 1954 & 94.0 & 1965 & 203.3 \\
1933 & 91.2 & 1944 & 151.2 & 1955 & 155.2 & 1966 & 54.4 \\
1934 & 158.0 & 1945 & 159.4 & 1956 & 334.1 & 1967 & 256.6 \\
1935 & 481.4 & 1946 & 339.8 & 1957 & 145.8 & 1968 & 87.2 \\
1936 & 190.9 & 1947 & 112.7 & 1958 & 94.9 & 1969 & 76.5 \\
1937 & 370.4 & 1948 & 173.6 & 1959 & 68.0 & 1970 & 122.6 \\
1938 & 120.9 & 1949 & 134.2 & 1960 & 41.3 & 1971 & 143.8 \\
1939 & 168.2 & 1950 & 279.8 & 1961 & 106.8 & 1972 & 339.8 \\
1940 & 47.6 & 1951 & 148.1 & 1962 & 211.8 & 1973 & 216.3 \\
\hline
\end{tabular}

Tabla 3. Gastos históricos y sistemáticos en $\mathrm{m}^{3} / \mathrm{s}$ del Río Irwell en Adelphi Weir, Inglaterra. (NERC, 1975)

\begin{tabular}{cc|cc|cc|cc}
\hline Año & Gasto & Año & Gasto & Año & Gasto & Año & Gasto \\
\hline 1896 & 311 & 1936 & 377 & 1947 & 272 & 1958 & 114 \\
1901 & 396 & 1937 & 100 & 1948 & 102 & 1959 & 176 \\
1908 & 255 & 1938 & 230 & 1949 & 292 & 1960 & - \\
1911 & 287 & 1939 & 186 & 1950 & 111 & 1961 & 252 \\
1919 & 348 & 1940 & - & 1951 & 230 & 1962 & 257 \\
1921 & 311 & 1941 & 205 & 1952 & 186 & 1963 & 288 \\
1923 & 289 & 1942 & 186 & 1953 & 378 & 1964 & 137 \\
1924 & 289 & 1943 & 246 & 1954 & 216 & 1965 & 320 \\
1927 & 340 & 1944 & 249 & 1955 & 295 & 1966 & 238 \\
1931 & 328 & 1945 & 496 & 1956 & 152 & 1967 & 287 \\
- & - & 1946 & 101 & 1957 & 278 & 1968 & 272 \\
\hline
\end{tabular}

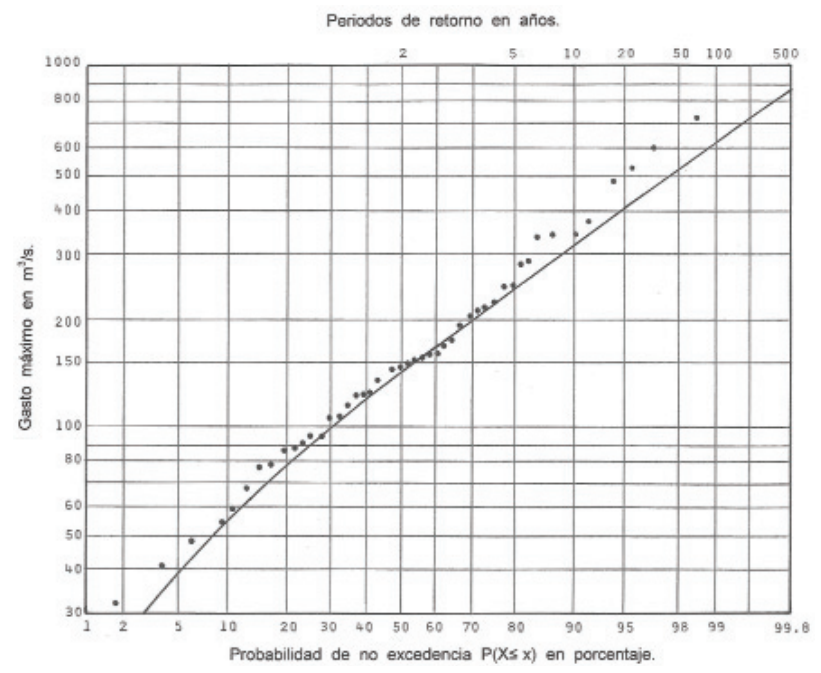

Figura 1. Contraste gráfico de la distribución log-normal de tres parámetros ajustada por momentos ponderados históricamente a los datos del Rio Big Sandy en Brucenton, USA (WRC, 1977).

Tabla 4. Gastos históricos y sistemáticos en $\mathrm{m}^{3}$ /s del Río Avon en Bath, Inglaterra. (Leese, 1974)

\begin{tabular}{cc|cc|cc|cc}
\hline Año & Gasto & Año & Gasto & Año & Gasto & Año & Gasto \\
\hline 1865 & 206 & 1940 & 148 & 1950 & 229 & 1960 & 352 \\
1866 & 228 & 1941 & 84 & 1951 & 136 & 1961 & 121 \\
1875 & 218 & 1942 & 149 & 1952 & 116 & 1962 & 103 \\
1879 & 264 & 1943 & 73 & 1953 & 96 & 1963 & 277 \\
1882 & 362 & 1944 & 118 & 1954 & 296 & 1964 & 110 \\
1888 & 204 & 1945 & 128 & 1955 & 128 & 1965 & 178 \\
1894 & 375 & 1946 & 282 & 1956 & 107 & 1966 & 172 \\
1899 & 239 & 1947 & 98 & 1957 & 138 & 1967 & 311 \\
1900 & 302 & 1948 & 104 & 1958 & 169 & 1968 & 125 \\
1924 & 255 & 1949 & 113 & 1959 & 169 & - & - \\
\hline
\end{tabular}


DOI: http://dx.doi.org/10.22201/fi.25940732e.2011.12n1.002

Campos-Aranda D.F.

Tabla 5. Valores característicos y predicciones $\left(\mathrm{m}^{3} / \mathrm{s}\right)$ en los cuatro ríos indicados

\begin{tabular}{|c|c|c|c|c|c|c|c|c|c|}
\hline \multirow{2}{*}{\multicolumn{2}{|c|}{$\begin{array}{l}\text { Variable Hidrológica* } \\
A N I H\end{array}$}} & \multicolumn{2}{|c|}{ Río Júcar, España } & \multicolumn{2}{|c|}{ Río Big Sandy, U.S.A. } & \multicolumn{2}{|c|}{ Río Irwell, Inglaterra. } & \multicolumn{2}{|c|}{ Río Avon, Inglaterra. } \\
\hline & & & 1792 & & 1897 & & 1896 & & 1865 \\
\hline & ANIS & & 1946 & & 1930 & & 1936 & & 1940 \\
\hline & ANFS & & 1988 & & 1973 & & 1968 & & 1968 \\
\hline & $Y t$ (años) & & 197 & & 77 & & 73 & & 104 \\
\hline & $N A H$ & & 4 & & 3 & & 10 & & 10 \\
\hline & $n(n c f)$ & & $42(1)$ & & $44(0)$ & & $31(2)$ & & $29(0)$ \\
\hline & $X c\left(\mathrm{~m}^{3} / \mathrm{s}\right)$ & & 6,200 & & 300 & & 240 & & 200 \\
\hline & na & & 4 & & 8 & & 25 & & 16 \\
\hline & $n b$ & & 42 & & 39 & & 16 & & 23 \\
\hline & $\hat{x}:\left(\mathrm{m}^{3} / \mathrm{s}\right)$ & & 732.886 & & 172.167 & & 215.384 & & 148.371 \\
\hline & $\hat{s}:\left(\mathrm{m}^{3} / \mathrm{s}\right)$ & & $1,716.725$ & & 124.701 & & 85.140 & & 63.655 \\
\hline & $\hat{g}$ & & 4.6913 & & 2.1007 & & 0.6074 & & 1.7445 \\
\hline & $c$ & & 1.1091 & & 0.6206 & & 0.1998 & & 0.5315 \\
\hline & $\alpha\left(\mathrm{m}^{3} / \mathrm{s}\right)$ & & -815.026 & & -28.776 & & -210.749 & & 28.599 \\
\hline & $\mu\left(\mathrm{m}^{3} / \mathrm{s}\right)$ & & 6.944 & & 5.140 & & 6.035 & & 4.661 \\
\hline & $\sigma\left(\mathrm{m}^{3} / \mathrm{s}\right)$ & & 0.896 & & 0.571 & & 0.198 & & 0.499 \\
\hline & Distribución ${ }^{* *}$ & LN3 & LP3 & LN3 & LP3 & LN3 & LP3 & LN3 & LP3 \\
\hline & $\operatorname{Tr}=2$ años & 222 & 110 & 142 & 142 & 207 & 224 & 134 & 138 \\
\hline & $\operatorname{Tr}=5$ años & 1387 & 765 & 247 & 234 & 283 & 307 & 190 & 201 \\
\hline & $\operatorname{Tr}=10$ años & 2452 & 1712 & 326 & 299 & 328 & 356 & 229 & 254 \\
\hline & $\operatorname{Tr}=25$ años & 4158 & 3501 & 435 & 387 & 380 & 410 & 282 & 335 \\
\hline & $\operatorname{Tr}=50$ años & 5709 & 5168 & 523 & 454 & 417 & 447 & 323 & 408 \\
\hline & $\operatorname{Tr}=100$ años & 7513 & 7013 & 616 & 522 & 451 & 480 & 366 & 493 \\
\hline & $\operatorname{Tr}=500$ años & 12835 & 11553 & 854 & 687 & 528 & 548 & 473 & 750 \\
\hline & $\operatorname{Tr}=1,000$ años & 15689 & 13469 & 968 & 760 & 559 & 574 & 523 & 892 \\
\hline & $\operatorname{Tr}=5,000$ años & 23875 & 17524 & 1259 & 938 & 631 & 629 & 647 & 1323 \\
\hline & $\operatorname{Tr}=10,000$ años & 28162 & 19046 & 1398 & 1017 & 661 & 650 & 705 & 1563 \\
\hline$* *$ & $\begin{array}{ll}\text { ANIH } & \text { año Inicial de las } \\
\text { ANIS } & \text { año Inicial de las } \\
\text { ANFS } & \text { año Final de las cr } \\
n c f & \text { número de crecier } \\
\text { LN3 } & \text { log-normal de } 3 p \\
\text { LP3 } & \text { log-Pearson tipo I }\end{array}$ & $\begin{array}{l}\text { s Históri } \\
\text { s Sistemá } \\
\text { Sistemát } \\
\text { intes en e } \\
\text { os. }\end{array}$ & $\begin{array}{l}\text { s. } \\
\text { cas. } \\
\text { as. } \\
\text { egistro sister }\end{array}$ & & & & & & \\
\hline
\end{tabular}

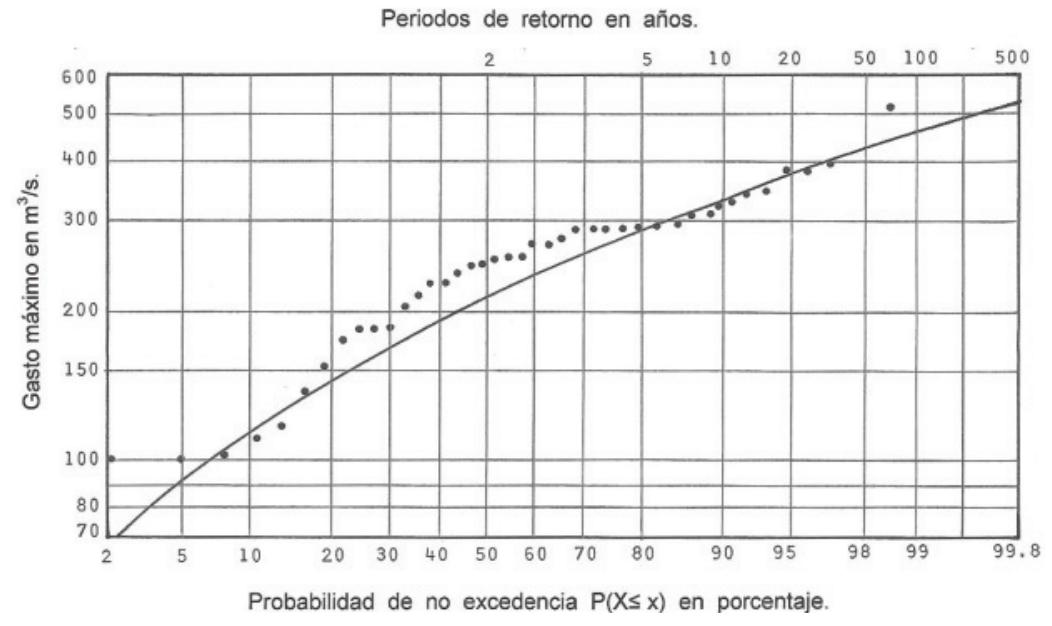

Figura 2. Contraste gráfico de la distribución log-normal de tres parámetros ajustada por momentos ponderados históricamente a los datos del Rio Irwell en Adelphi Weir, Inglaterra (NERC, 1975). 


\section{Conclusión}

El método de los momentos ponderados históricamente (mph), es quizás el enfoque más simple que permite incorporar la información sobre crecientes históricas disponible en algunas localidades. El uso de los mph para ajustar la distribución Log-normal de 3 parámetros que representa tales datos, es también un procedimiento sencillo y eficaz, al juzgar por los resultados numéricos obtenidos en las aplicaciones descritas y en los contrastes gráficos expuestos. Por lo anterior, se recomienda su aplicación como procedimiento inicial, cuando se deban procesar probabilísticamente registros sistemáticos que disponen de información histórica sobre crecientes.

\section{Referencias}

Abramowitz M., Stegun I.A. Handbook of Mathematical Functions. Chapter 3: Elementary Analytical Methods, pp. 9-63 and chapter 26. Probability functions, pp. 925-995. Ninth Printing. New York, U.S.A. Dover Publications, Inc. 1972. 1046 p.

Benson M.A. Measurement of Peak Discharge by Indirect Methods. Technical Note. 90 (WMO-No. 225). World Meteorological Organization. Geneva, Switzerland. 1968. 161 p.

Bobée B. The Log Pearson Type 3 Distribution and its Application in Hydrology. Water Resources Research, 11(5):681-689. 1975.

Campos-Aranda D.F. Análisis probabilístico univariado de datos hidrológicos. Capítulo 1: Conceptos teóricos del análisis probabilístico, pp. 11-31. Avances en Hidráulica 13. AMH-IMTA. México DF. 2006. 172 p.
Condie R., Lee K.A. Flood Frequency Analysis with Historic Information. Journal of Hydrology, 58:47-61. 1982.

Francés G.F. Utilización de la información histórica en el análisis regional de las avenidas. Capítulo 6: Aplicación a los Ríos Júcar, Turia y Segura, pp. 166-213. Monografía No. 27 del Centro Internacional de Métodos Numéricos en Ingeniería. Barcelona, España. 1995. 242 p.

Hirsh R.M., Stedinger J.R. Plotting Positions for Historical Floods and their Precision. Water Resources Research, 23(4):715-727. 1987.

Instituto Mexicano de Tecnología del Agua. Banco Nacional de Datos de Aguas Superficiales (BANDAS). Hidrometría y sedimentos hasta 2002 en 8 CD's. SEMARNAT-CNA-IMTA. Jiutepec, Morelos. 2003.

Kite G.W. Frequency and Risk Analyses in Hydrology. Chapter 12: Comparison of Frequency Distributions, pp. 156-168. Water Resources Publications. Fort Collins, Colorado, USA. 1977. 224 p.

Leese M.N. The Use of Censored Data in Estimating T-Year Floods. Design of Water Resources Projects with Inadequate Data, pp. 563-575. Proceedings of the Madrid Symposium, June 1973. Studies and Reports in Hydrology 16. UNESCO-WMOIAHS. Paris, France. 1974.

Natural Environment Research Council. Flood Studies Report. Volume I (Hydrological Studies), chapter 2, theme 2.8: The Treatment of Missing Peaks or Historic Floods as Censored Samples, pp. 213-219. NERC. London, England. 1975.

Tasker G.D., Thomas W.O. Flood-Frequency Analyses with Prerecord Information. Journal of the Hydraulics Division, 104(HY2): 249-259. 1978.

Water Resources Council. Guidelines for Determining Flood Flow Frequency. Appendix 6: Historic data, pp. 6.1-6.7. Bulletin 17 A of the Hydrology Committee. WRC. Washington, D. C., U.S.A. 1977. Revised edition. 1977.

\section{Semblanza del autor}

Daniel Francisco Campos-Aranda. Obtuvo el título de ingeniero civil en diciembre de 1972 en la entonces Escuela de Ingeniería de la Universidad Autónoma de San Luis Potosí. Durante el primer semestre de 1977, realizó en Madrid, España un diplomado en hidrología general y aplicada. Posteriormente, durante 1980-1981, llevó a cabo estudios de maestría en ingeniería en la especialidad de hidráulica en la División de Estudios de Posgrado de la Facultad de Ingeniería de la UNAM. En esta misma institución, inició (1984) y concluyó (1987) el doctorado en ingeniería con especialidad en aprovechamientos hidráulicos. Ha publicado artículos principalmente en revistas mexicanas de excelencia: 35 en Ingeniería Hidráulica en México, 11 en Agrociencia y 6 en Ingeniería. Investigación y Tecnología. En congresos internacionales y nacionales ha presentado 24 y 73 ponencias, respectivamente. Fue investigador nacional (nivel I: expediente 7273) desde el $1^{\circ}$ de julio de 1991 hasta el 31 de diciembre del 2007. Actualmente es profesor jubilado de la UASLP, desde el $1^{\circ}$ de febrero del 2003. En 2008, la AMH le otorgó el premio nacional "Francisco Torres H.", a la práctica profesional de la hidráulica. 